\title{
Improving Timeliness of Georgia Emergency Room Data
}

\author{
Lance S. Ballester*, Karl Soetebier, Bill Williamson, Rene Borroto, Jessica Grippo, \\ Patrick Pitcher and Cherie Drenzek
}

Georgia Department of Public Health, Atlanta, GA, USA

\section{Objective}

To explore the timeliness of emergency room surveillance data after the advent of federal Meaningful Use initiatives and determine potential areas for improvement.

\section{Introduction}

Timeliness of emergency room (ER) data is arguably its strongest attribute in terms of its contribution to disease surveillance. Timely data analyses may improve the efficacy of prevention and control measures.

There are a number of studies that have looked at timeliness prior to the advent of Meaningful Use, and these studies note that ER data were not fast enough for them to be useful in real time ${ }^{2,3}$. However, the change in messaging practices in the Meaningful Use era potentially changes this.

Other studies have shown that changes in processes and protocol can dramatically improve timeliness ${ }^{1,4}$ and this motivates the current study of timeliness to identify processes that can be changed to improve timeliness.

\section{Methods}

ER data were collected from March 2017 through September 2017 from both the Georgia Department of Public Health's (GDPH) State Electronic Notifiable Disease Surveillance System (SendSS) Syndromic Surveillance Module and the Centers for Disease Control and Prevention (CDC) National Syndromic Surveillance Program's (NSSP) ESSENCE systems. Patients from hospitals missing 10 or more days of data, as well as patients with missing or invalid triage times, and all visits after August 1st were excluded in order to ensure data were representative of a "typical" time period and that a sufficient amount of time was given for visits to arrive from hospitals.

The timeliness of individual records was determined in a number of different ways. All timeliness measurements were determined by subtracting the earlier time event from the later time of the event. The overall measure of timeliness is the time between the patient's triage time and the data being present in the ESSENCE data system. In between, Georgia's SendSS system receives and processes the data. This is illustrated in Figure 1. Due to the skewed nature of these measures, they were analyzed using medians and Gaussian kernel density plots.

\section{Results}

The study in total included records from 118 Georgia hospitals, 14,203 data files and 1,897,501 patient records. Overall median timeliness of data from Triage Time to being available in SendSS for analyses was 33.62 hours (IQR=28.5), and in ESSENCE was 45.08 hours (IQR=37.05).

The distributions of Triage Time of Day, Time Available in SendSS Staging, and Time Available in ESSENCE Analysis can be seen in Figure 2. Additionally, lines were added for when SendSS makes data available for its own analyses and when it sends data to ESSENCE. These latter lines represent places where the SendSS system itself could improve, and potential improved times were noted based on the kernel densities.

Peak triage times for Georgia hospitals were between 10 am to 11 pm, shown in black. This represents the ideal timeliness if hospitals sent their data immediately. However, data was all batched by Georgia hospitals and sent at different times of the day. The distribution of the time patient records arrived at SendSS staging was indicated in blue.

During the period of this study, Georgia processed data into its SendSS system at 6:30am and 11:30am every day and sent data to the ESSENCE system at $1 \mathrm{pm}$ each day. These times are highlighted on the plot in green, and red respectively. New potential improved times, based on the kernel density of data being available in SendSS staging, are shown in the lighter shades of these colors at 8:30am and $12 \mathrm{pm}$ every day, while being sent to ESSENCE at 9am and 12:30pm to ensure time for data to be properly processed. These were determined to be optimal times for reducing lag in the data, however, may not be optimal for daily analysis.

The purple line on the plot represents the times that data were available in ESSENCE's system for analysis. This was notably delayed by a median 4.15 hours after the data was sent to ESSENCE on a typical day.

\section{Conclusions}

A data driven approach to choosing processing times could improve timeliness of data analyses in the SendSS and ESSENCE systems. By conducting this type of analysis in an ongoing periodic basis, processing lag times can be kept at a minimum.

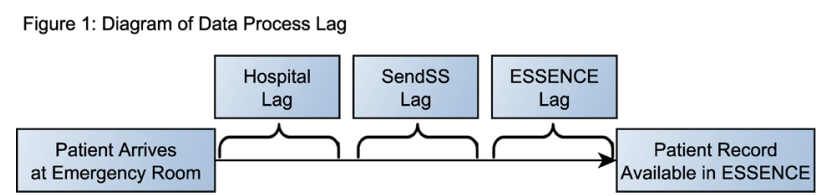

Figure 2: Gaussian Kernel Densities of Data Process Times

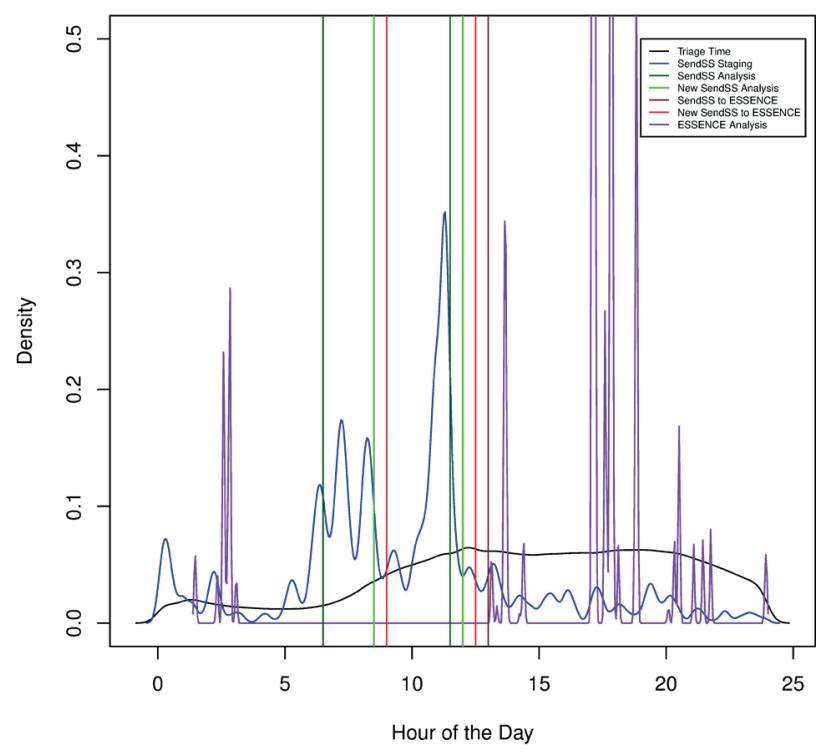




\section{ISDS 2018 Conference Abstracts}

\section{Keywords}

Timeliness; Meaningful Use; ESSENCE

\section{References}

1. Centers for Disease Control. Progress in improving state and local disease surveillance--United States, 2000-2005. MMWR Morbidity and mortality weekly report. 2005;54(33):822-825.

2. Jajosky R, Groseclose S. Evaluation of reporting timeliness of public health surveillance systems for infectious diseases. BMC Public Health. 2004;4(1)

3. Travers D, Barnett C, Ising A, Waller A. Timeliness of emergency department diagnoses for syndromic surveillance. AMIA Annual Symposium Proceedings. 2006; Vol. 2006:769.

4. Ward M, Brandsema P, van Straten E, Bosman A. Electronic reporting improves timeliness and completeness of infectious disease notification, The Netherlands, 2003. Eurosurveillance. 2005;10(1):7-8.

\section{*Lance S. Ballester}

E-mail: lanceballester@gmail.com 